\title{
Haptic Soft-Keyboard for Tablet-Sized Touchscreens
}

\author{
Sunjin Yu ${ }^{1}$, Sung Hun Jin ${ }^{2}$ and Kwangtaek Kim ${ }^{3, *}$ \\ 1 School of Digital Media Engineering, Tongmyong University, Busan 48520, Korea \\ 2 Department of Electronics Engineering, Incheon National University, Incheon 22012, Korea \\ 3 Department of Information and Telecommunication Engineering, Incheon National University, \\ Incheon 22012, Korea \\ * Correspondence: ktkim@inu.ac.kr; Tel.: +82-835-8628
}

Received: 4 June 2019; Accepted: 26 July 2019; Published: 31 July 2019

\begin{abstract}
Most users are not satisfied with the typing vibration feedback from linear motors of mobile phones since it feels like buzzing rather than physical key pressing feedback. For larger touchscreens, such as 10.1 inch tablets, there is no commercial tactile soft keyboard developed yet. Therefore, there is the need to develop a haptic soft keyboard technology imitating the physical keyboard for the tablet-sized touchscreen. In the present study, we present a new approach introducing an optimal design of a multilayered piezoelectric actuator that meets the need for a haptic soft keyboard for 10.1 inch tablets, implementing stronger tactile feedback that utilizes human sensitivity to time differences obtained by a psychophysical experiment and equalizes the force distribution of the touchscreen when using multiple piezoelectric actuators. The developed system was evaluated by a user study measuring typing performance.
\end{abstract}

Keywords: haptic feedback; soft keyboard; mobile input device; user interface; haptic soft keyboard; tablet touchscreen; tactile perception

\section{Introduction}

These days, the soft keyboard is one of the most used input methods in devices from mobile phones to tablets. Researchers have been working to invent or develop advanced typing methods for mobile touchscreens for over a decade. Most of the efforts were put forth to propose new layouts of key characters by analyzing users' typing behaviors or to improve typing productivity using add-on methods such as typing error correction and autocomplete. However, most of the frustration users feel is due to there being no real key click feedback when typing on the touchscreen. For this, phone users tend to use synchronized vibrations generated from a low-cost linear motor when they type. Nevertheless, most of the users are not satisfied with the built-in vibration feedback of mobile phones, since it feels like buzzing rather than physical key pressing feedback. For larger touchscreens, such as 10.1 inch tablets, there is no commercial tactile soft keyboard available yet. Therefore, there is a need to develop a haptic soft keyboard technology that imitates the physical keyboard for larger touchscreens of mobile devices, including tablets.

To this end, piezoelectric actuators that have fast response time (10 to $50 \mu \mathrm{s})$ and strong deformation (even up to a few hundred millimeters when high voltage is applied) were introduced to be mounted inside touchscreens [1,2]. Despite the strength of piezoelectric actuators, there is little known about how to use them for implementing tactile key click effects on commercial tablet-sized touchscreens, because piezoelectric actuators generally require very high voltage to be deformed. For instance, single layered piezoelectric actuators are operated at 100 to 200 volts (peak to peak). The required voltage can be reduced by designing multilayered piezoelectric actuators; however, due to the capacitance, the input current should be increased proportional to the number of internal layers. Concerning the need for 
stronger tactile feedback for larger mobile devices such as tablets, multilayer piezoelectric actuators are preferred to implement tactile feedback on touchscreens. To provide tactile key click effects on the entire touchscreen of a 10.1 inch tablet, multiple piezoelectric actuators should be embedded and controlled accurately using a mobile battery. However, driving multiple piezoelectric actuators at the same time on the touchscreen of a tablet is a challenging issue.

The first prototype of a haptic soft keyboard using four piezoelectric actuators was developed with two commercial mobile devices (a Windows phone and Microsoft Surface Pro tablet) by Han and Kim [1]. They used a portable piezoelectric driver that can drive up to four multilayered piezoelectric actuators at the same time. For the haptic soft keyboard of the tablet, they developed the haptic soft keyboard and implemented an add-on interface for tactile feedback to the touchscreen. A user study measuring the performance of key typing on the touchscreen for three feedback conditions (visual only, visual-aural, and visual-haptic) was conducted, and the results showed improved key typing performance with haptic feedback in terms of accuracy, speed, and usability. They showed how to utilize multiple piezoelectric actuators to simulate a seemingly real key click effect on a larger touchscreen. However, there was a $10 \mathrm{~ms}$ time delay to get tactile feedback, the multilayer piezoelectric actuators were too thick, and the piezo driver circuit was too big to be embedded. In a following study, Kim developed a standalone haptic soft keyboard that can be simply added to existing tablet devices regardless of operating systems [2].

In the present study, we present a new approach introducing an optimal design for a multilayered piezoelectric actuator that meets the need of a haptic soft keyboard for 10.1 inch tablets, implementing stronger tactile feedback by utilizing human sensitivity to time differences obtained by a psychophysical experiment and equalizing the force distribution of the touchscreen. This paper is organized as follows. Section 2 introduces related studies that deal with new designs of the soft keyboard by analyzing users' typing behaviors or adding tactile feedback. Section 3 presents how we found the optimal parameters and designed multilayer piezoelectric actuators needed for 10.1 inch touchscreens. Additionally, a perception study to estimate human sensitivity to time differences on touchscreens and an equalization algorithm for uniform force distributions are described. Experimental results of the quantitative evaluation of the developed piezoelectric actuators and force distribution method and the results of a user study measuring typing performance with the developed haptic soft keyboard are reported in Section 4. Finally, discussions are provided in Section 5.

\section{Related Work}

Early studies focused on analyzing typing fingers and key typing patterns on touchscreens to improve existing soft keyboard designs. Findlater et al. designed a new QWERTY layout of a soft keyboard by adopting a typist's typing patterns $[3,4]$. They conducted experiments to analyze typing positions and postures on touchscreens over a period of time and applied the results for a personalized layout. Their studies showed that a personalized layout based on typing patterns and behaviors significantly improved typing speed on the touchscreen of mobile devices. Similarly, Li et al. designed a QWERTY layout in a single line that consists of eight buttons and recognizes the user's intention of the text input from typing finger postures [5]. The results demonstrated that users quickly learn the new text entry method. In line with this approach, Goel et al. proposed a new text entry method utilizing the user's finger postures and motions to improve typing performance on touchscreens [6]. Most recently, Jo and Cho proposed a new method using random forest that automatically recommends a preferred layout for the user by adopting trained data of typing behaviors and patterns [7]. Due to all of these studies, the usability of the soft keyboard on touchscreens has been greatly improved.

Unlike the physical keyboard, it is hard to confirm that a key was pressed or clicked on flat touchscreens. A simple way of confirming a pressed key is to change the color of the typed key character or to provide a key-click sound effect. However, users are not satisfied with these methods and prefer to feel a tactile key-click effect as they do with the physical keyboard. To add a tactile key effect, Poupyrev and Maruyama designed and implemented a tactile feedback interface for a Personal 
Digital Assistant (PDA) touchscreen [8]. They conducted a user study with ten participants to prove the usability of the tactile feedback in comparison with audio feedback. Their results demonstrated the effectiveness of tactile feedback on the touchscreen of a PDA. Rabin and Gordon studied a similar study but for a medical application [9]. They showed that tactile feedback plays an important role in typing by letting users know the start position without looking at the touchscreen. From a practical perspective, Brewster et al. studied the effectiveness of vibro-tactile feedback using a commercial vibrator attached to the backside of a mobile device [10]. The results showed that typing speed and accuracy were improved through the tactile feedback generated from the backside. Hoggan et al. showed the same result for text typing on a touchscreen [11].

Koskinen et al. studied pleasant feelings on touchscreens with two different actuators, piezoelectric actuators and linear motors [12]. Their study showed that users feel more pleasant with piezoelectric actuators. Jansen et al. developed the MudPad, which is capable of localized haptic feedback on multi-touch surfaces [13]. Hoffman et al. demonstrated that tactile feedback can also be used for preventing typing errors [14], and McAdam et al. investigated tactile feedback on text entry with tabletop computers. In their study, tactile feedback improved typing speed, even though actuators were attached to users' bodies [15]. Kaaresoja at al. showed that the latency of touch feedback did not significantly affect typing performance on the touchscreen [16]. In regards to developing a prototype with multiple piezoelectric actuators, Han and Kim showed the possibility of completely embedding the actuators into the commercial mobile devices (phones and tablets) [4,17]. Their study showed how to drive multiple piezoelectric actuators on a commercial tablet for the first time and the effectiveness of a tactile key click effect on touchscreens by conducting user studies. However, the prototype had a time delay (over $10 \mathrm{~ms}$ ), which resulted in delayed tactile feedback to fast typing. Kim extended the approach by developing a standalone haptic soft keyboard system using a microcontroller [2]. Most recently, Jeon et al. studied the effectiveness of tactile feedback on the virtual keyboard [18].

\section{Materials and Methods}

In this section, an optimal design of piezoelectric actuators, a perception study measuring discrimination thresholds on time differences of a tactile signal on touchscreen, and an algorithm that globally equalizes force distributions on touchscreens are presented.

\subsection{Design of Haptic Actuators for Tablet Touchscreens}

\subsubsection{Piezoelectric Ceramic Actuators}

The piezoelectric effect is to generate an electric charge in response to mechanical stress, and its inverse effect refers to mechanical deformation when an electric field is applied in the polarization direction of piezoelectric materials. These effects can be used as both sensors and actuators. In this study, we focus on the inverse piezoelectric effect, which is the case for piezoelectric actuators. Fundamental equations for dielectrics can be written as:

$$
\begin{aligned}
& C=\epsilon \frac{A}{t} \\
& Q=C V
\end{aligned}
$$

where $A, t$, and $\epsilon$ denote the area of the capacitor plate, the thickness of the plate, and the dielectric constant, and $Q, C$, and $V$ are charge, capacitance, and voltage applied, respectively. Then, (2) can be rewritten by (1):

$$
Q=\frac{\epsilon A V}{t}
$$


Additionally, the mechanical displacement $\delta$, which can be defined as charge density or the ratio of charge to the area of the capacitor is obtained as follows:

$$
\delta=\frac{Q}{A}=\frac{\epsilon V}{t}
$$

Further, $\delta$ can be derived as a simplified form for two layered and three layered actuators from [19]:

$$
\begin{gathered}
\delta=\frac{3 d_{31} l^{2} V}{t^{2}} \\
\delta=\frac{6 S_{11}^{m} d_{31}\left(t_{m}+t_{p}\right) l^{2} V}{2 S_{11}^{m}\left(3 t_{m}^{2} t_{p}+6 t_{m} t_{p}^{2}+4 t_{p}^{3}\right)+S_{11}^{E} t_{m}^{3}}
\end{gathered}
$$

where $S_{11}^{m}$ is the elastic coefficient of the supporting layer $\left(\mathrm{m}^{2} / \mathrm{N}\right), S_{11}^{E}$ is the elastic coefficient of the piezoelectric layer $\left(\mathrm{m}^{2} / \mathrm{N}\right), t_{m}$ is the thickness of the supporting layer $(\mathrm{mm}), t_{p}$ is the thickness of the piezoelectric layer $(\mathrm{mm}), l$ is the length of the piezo, and $d_{31}$ is the piezoelectric coefficient $(\mathrm{mm} / \mathrm{V})$.

\subsubsection{Optimal Design of Piezoelectric Benders}

In this study, we focus on maximizing the mechanical displacement $\delta$ while meeting the size requirements (thickness less than $0.5 \mathrm{~mm}$ and width of $8 \mathrm{~mm}$ ). To maximize the displacement $\delta$ assuming the material coefficients and the applied voltage to be constant, the layer thickness should be optimized by controlling the number of layers and the layer ratio. Thus, two factors (layers and the ratio of layers) were investigated by simulations, and then the structure of a piezoelectric bender to be mounted underneath the tablet touchscreen was designed and manufactured. There is limited space on the bezel of tablet touchscreens; thus, the width and the thickness of the actuator were fixed to $8 \mathrm{~mm}$ and $0.4 \mathrm{~mm}$, respectively, due to the space limit [10 $\mathrm{mm}$ width on the bezel and a $0.5 \mathrm{~mm}$ gap between the touchscreen and the liquid crystal display (LCD) panel] of commonly used 10.1 inch tablets). A software tool, COMSOL Multiphysics, using a finite element method was used for simulating the bending effects of piezoelectric actuators from a single layer (called unimorph) to three layers. Our intention with the tool is to simulate the behavior of piezo bending in the $\mathrm{z}$ axis as a square wave $(500 \mathrm{~Hz}, 20 \mathrm{Vpp})$ is applied, which will generate a force under the touchscreen that is eventually bent. A rectangle plate $[(\mathrm{H}) 40 \times(\mathrm{W}) 8 \times(\mathrm{T}) 0.4 \mathrm{~mm}]$ was designed and simulated with parameters [pole directions: down (unimorph); up and down (bimorph); up-up-down (three layers), layer thickness: $0.3 \mathrm{~mm}$ (unimorph); $0.15 \mathrm{~mm}$ each (bimorph); $0.1 \mathrm{~mm}$ and $0.14 \mathrm{~mm}$ (three layers) for $\mathrm{Pe}$ and $\mathrm{Pi}$, respectively]. The pole directions were found by a pilot analysis investigating the bending strength versus pole directions (in series or parallel). The simulation was run on a Windows machine (CPU i5-6500 3.2 Hz, RAM 8 GB, Graphic GTX 1050).

Figure 1 shows the results of simulating piezoelectric benders driven by a square wave $(500 \mathrm{~Hz}$, $20 \mathrm{Vpp}$ ). The magnitude of displacement increased as the number of piezo's layers increased. The displacement of the three-layer bender was three times greater than that of the two-layer bender in the series pole direction, which justified the need for three layers. Furthermore, the simulation indicated that the ratio of the three layers could affect the displacement performance. Thus, the other simulation was conducted to figure out the optimum ratio value. For the simulation, three layers were defined as Pe (external layer) and $\mathrm{Pi}$ (internal layer), and the ratio was computed by $\mathrm{Pi} / \mathrm{Pe}$. The right plot of Figure 1 shows that the max displacement of the bender was obtained at 1.4 (i.e., $\mathrm{Pi}=0.14 \mathrm{~mm}$ and $\mathrm{Pe}=0.1 \mathrm{~mm}$ ), which demonstrates that the thickness of the internal ceramic layer (Pi) significantly affected the bending performance of the piezo bender. Based on the simulations, a final design of piezoelectric actuators (three layers and 1.4 as its ratio) was determined, as seen in Figure 2. The estimated max displacement was $600 \mu \mathrm{m}$ at $100 \mathrm{Vpp}$. 

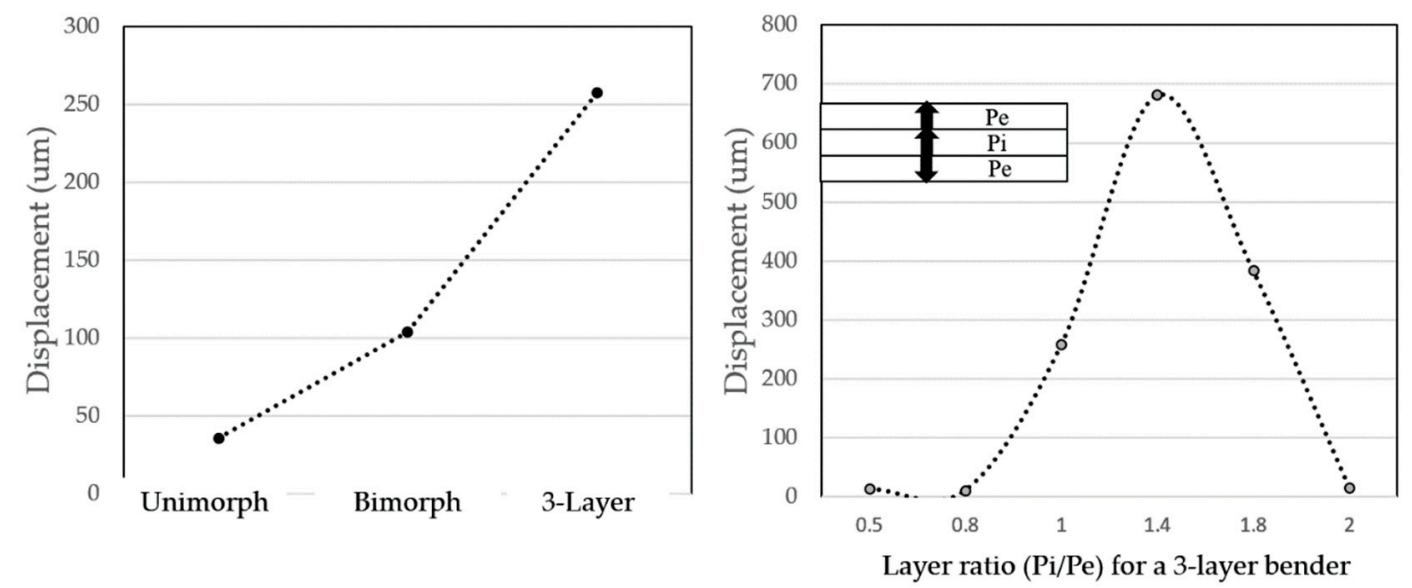

Figure 1. Results of bending simulations for a piezoelectric plate $[(\mathrm{H}) 40 \times(\mathrm{W}) 8 \times(\mathrm{T}) 0.4 \mathrm{~mm})]$ with COMSOL Multiphysics: displacement $(\mu \mathrm{m})$ in the $\mathrm{z}$ axis from a single layer to three layers (left) and the optimal ratio (Pi/Pe) for a three-layer bender to be bent most (right). A square wave (500 Hz, $20 \mathrm{Vpp}$ ) was applied to the piezo plate for simulations.

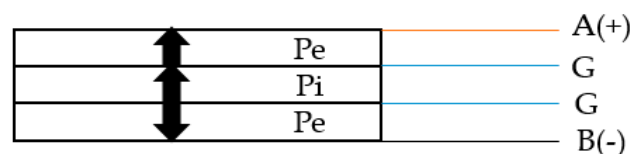

(a)

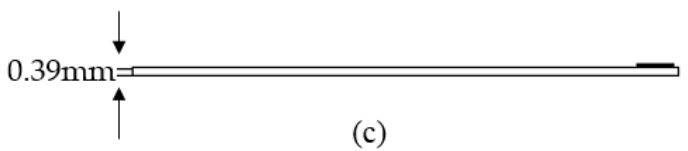

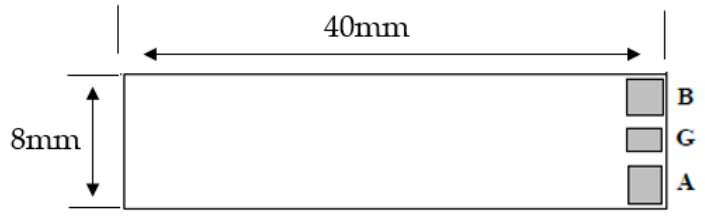

(b)

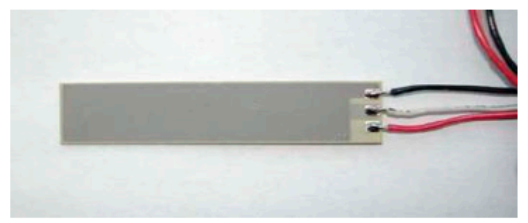

(d)

Figure 2. Final design $(\mathbf{a}-\mathbf{c})$ of a three-layer piezoelectric actuator based on the results of simulations and $(\mathbf{d})$ is a manufactured actuator. Pe and Pi are $0.1 \mathrm{~mm}$ and $0.14 \mathrm{~mm}$, respectively. A and B are positive and negative electrodes, and $\mathrm{G}$ is the ground, respectively. The final thickness $(0.39 \mathrm{~mm})$ was estimated including bonding.

\subsection{Perception Study to Estimate the Sensitivity to Time Difference of Tactile Key Click}

Understanding the human perception of key click tactile feedback on touchscreens by piezoelectric actuators is a crucial step to designing the haptic soft keyboard due to the limited tablet power to consistently drive multiple piezoelectric actuators. Therefore, our strategic approach was to drive piezoelectric actuators more efficiently (saving power) while maximizing human perceptibility on touchscreens by utilizing temporal discrimination thresholds of the piezo bending effect. It was learned through a pilot experiment that humans perceive a stronger key click effect when subsequent double bending imperceptibly occurs. This double bending effect of piezoelectric actuators can be achieved by driving with a pulse train that has two peaks with an imperceptible interval, which should be smaller than a temporal discrimination threshold. To estimate the temporal touch discrimination thresholds on the touchscreen of a 10.1 inch tablet device (Samsung Galaxy Tab), a psychophysical experiment was designed and conducted using an adaptive psychophysical method for two conditions, haptics only and haptics combined with audio (key click sound with the same $500 \mathrm{~Hz}$ square wave). The combined condition was also tested to see the increased sensitivity by adding audible feedback, which is commonly used for typing on mobile touchscreens. 


\subsubsection{Participants}

A total of fifteen participants (eight males and seven females, age range 22-30, average age 26.4 years old) took part in the experiment. All participants were right-handed by self-report. Two participants had previous experience with haptic interfaces and perception experiments. None of the participants reported any deficiencies in hearing or touch.

\subsubsection{Stimuli}

Two stimuli, the test and the reference (see Figure $3 a, b$ ), were designed and used for the perception study. In the test pulse, the time interval $t$ (Figure 3a) was controlled to increase or decrease from trial to trial based on participants' responses during the experiment. For the reference pulse, the duration was fixed to $2 \mathrm{~ms}$ defined by the period of $500 \mathrm{~Hz}$. To drive piezoelectric actuators efficiently, a simple piezo driver circuit was developed using an 8 bit microcontroller (AtMega 128, $16 \mathrm{MHz}$ ) with an acoustic signal amplifier (TI DRV 2667, gain $38.4 \mathrm{~dB}$ ). As seen in Figure 4, test and reference pulses were generated in the microcontroller and amplified to $65 \mathrm{Vpp}$ with the amplifier and then sent to a piezoelectric actuator attached to the 10.1 inch touchscreen to simulate a tactile key click effect.

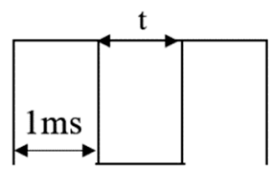

(a)

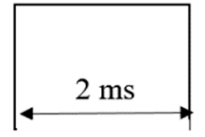

(b)

Figure 3. Two stimuli, (a) test and (b) reference, simulating a key click effect used for the psychophysical experiment. The time interval $(t)$ between two peaks was controlled during the experiment.

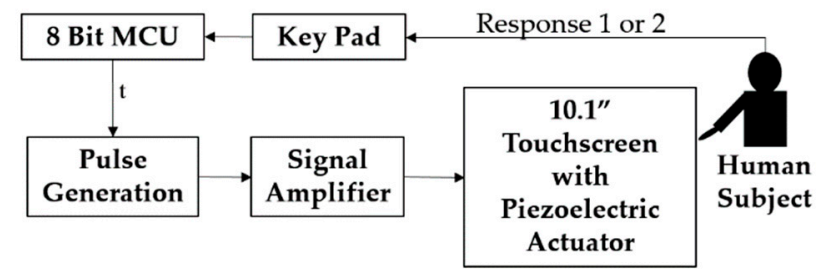

Figure 4. Experimental setup of a perception study measuring temporal discrimination thresholds on a 10.1 inch touchscreen. The participant passively felt tactile feedback on the touchscreen and then responded to the provided stimulus.

\subsubsection{Procedures}

A two-interval forced choice (2IFC) one-up one-down adaptive procedure [20] was used for the experiment to estimate the discrimination thresholds of the tactile key click effect simulated by a piezoelectric actuator designed in this study. Participants were tested with two conditions, haptics alone $(\mathrm{H})$ or haptics combined with audible sound (HA). On each trial, for the $\mathrm{H}$ (or the HA) condition, the participants put their index finger on the tablet touchscreen (Samsung Galaxy Tab) and felt the key click effect simulated by the piezoelectric actuator driven by the stimulus signal (a $500 \mathrm{~Hz}$ square wave signal). For the HA condition, the participants perceived the key click effect with the same square wave $(500 \mathrm{~Hz})$ through both the touchscreen and the headphone. The sound was generated with a sound play function implemented in the microprocessor. The reference stimulus was presented with a $2 \mathrm{~ms}$ square wave, while the test stimulus was controlled by a time interval $(t)$, as seen in Figure 3 . The reference and the test stimuli were randomly displayed to avoid subjects' expectation errors. The participant's task was to indicate which one was felt as two pulses. According to the one-up one-down adaptive rule, the value of $t$ in the test pulse was increased after an incorrect response and decreased after a correct response. The initial $t$ value was chosen to be large enough $(15 \mathrm{~ms})$ so that the two peaks of a test pulse train were clearly felt to the participant. The value of $t$ then decreased or increased by a 
step size of $6 \mathrm{~dB}$ depending on the participant's responses. After the initial three reversals, the value of $t$ changed by $2 \mathrm{~dB}$. The initial larger change in $t$ was necessary for fast convergence, whereas the later smaller change improved the accuracy of threshold estimates. Each adaptive series was terminated after eight reversals at the smaller step size. The participants were comfortably seated in front of the touchscreen with a keypad, as shown in Figure 4. They wore headphones to block any sound from the equipment for the $\mathrm{H}$ condition. Initial training was provided, where a series of stimuli were presented to familiarize the participants with the tactile key click effect on the touchscreen and the two experimental conditions ( $\mathrm{H}$ and $\mathrm{HA}$ ). The training time varied from participant to participant and averaged $10 \mathrm{~min}$. Each participant was tested six times per condition, resulting in a total of twelve adaptive series per participant. It took between forty minutes to one hour for each participant to complete all the 12 series.

\subsubsection{Data Analysis}

For each adaptive series, thresholds were calculated from the values over the last eight reversals at the $2 \mathrm{~dB}$ step size. Specifically, two thresholds were estimated by averaging the last eight reversals for the two conditions ( $\mathrm{H}, \mathrm{HA})$. The discrimination thresholds were equally considered "just noticeable discrimination" (JND) on temporally displayed tactile pulses on the touchscreen.

\subsubsection{Results of the Psychophysical Experiment}

Figure 5 shows the results of the psychophysical experiment that estimated temporal discrimination thresholds (3.96 ms for $\mathrm{H}$ and $2.27 \mathrm{~ms}$ for HA) of key clicks on a tablet touchscreen. The graph bars represent the thresholds for all participants for the two conditions, H and HA, and the error bars show standard deviations. In the left plot, it was observed that the thresholds for the $\mathrm{H}$ condition were all higher than those of the HA condition over all the participants. It was also apparent in the right that the average threshold for $\mathrm{H}$ was larger than that of HA. A one-way ANOVA with the condition $(\mathrm{H}, \mathrm{HA})$ showed that there was a significant difference between the two conditions $[F(1,118)=160.1$, $p<<0.0001]$. The results demonstrated that (1) a tactile display of sequential two peaks with a smaller interval than $3.96 \mathrm{~ms}$ could be perceived as one peak, and (2) the perception of tactile key clicks could be more sensitive by adding a synchronized audio cue, which can serve as a guideline in both sound and touch feedback on touchscreens. The touch sensitivity (about $4 \mathrm{~ms}$ ) to time difference measured in this study was slightly smaller than the $5 \mathrm{~ms}$ reported in Goldstein's book [21]. The increased touch sensitivity when combined with aural feedback was expected due to hearing dominance-the sensitivity to time difference was about $0.01 \mathrm{~ms}$ reported in [21]. In this study, the threshold estimated by the haptics only condition was adopted for the haptic soft keyboard.

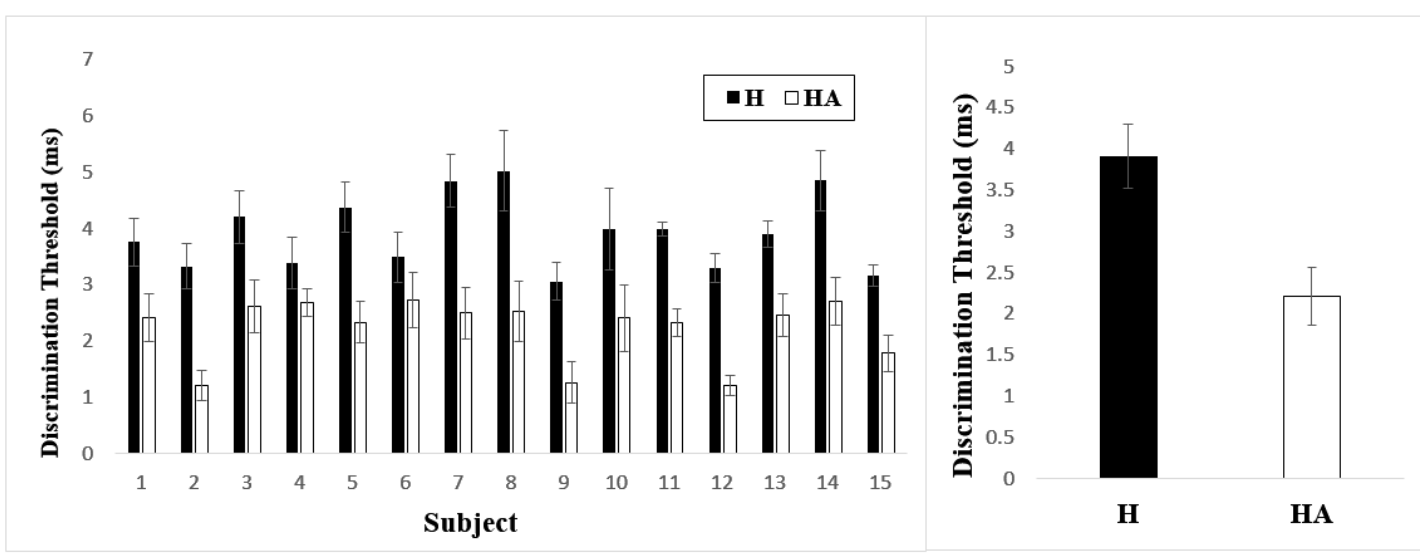

Figure 5. Discrimination thresholds (temporal sensitivity) estimated on tactile key click effects on tablet touchscreens: thresholds estimated per participant (left) and the average thresholds (right) for the haptics alone $(\mathrm{H})$ and haptics with audible sound (HA) conditions. 


\subsection{Global Equalization of Tactile Key Click on Tablet Sized Touchscreens}

Until now, the best designs of piezoelectric actuators and an imperceptible two-peak pulse were found to provide a strong bending effect within the required thickness $(0.4 \mathrm{~mm})$ and to efficiently maximize perceptual key click feedback on touchscreens. However, these findings are focused on increasing the strength of local tactile feedback. Equalizing force feedback on a touchscreen is a difficult problem because, in general, the strength of tactile feedback is too strong at the bezel but too weak at the center of the touchscreen. To globally equalize the strength of key click feedback on touchscreens, we propose a new algorithm that adaptively changes the strength of tactile feedback, as summarized in Figure 6. The strategy is to reduce strength on or near the bezel while keeping the maximum piezo bending at the weak area. As seen in Figure 6, tactile feedback (acceleration) on a touchscreen is proportional to the input voltage driven to the piezoelectric actuator. The linear relationship between acceleration and input voltage can be represented as a linear regression model found by line fitting (see the left of Figure 6). From the linear regression model, an inverse function is derived to estimate an input voltage value:

$$
V_{i}=\frac{G+0.21}{1.17}
$$

where $G$ is a desired acceleration value, and $V_{i}$ denotes an input voltage value to piezoelectric actuators to be controlled. In this study, $G$ was set to $1.5 \mathrm{G}$, which was above the detection threshold $(1.17 \mathrm{G})$ found by Kim [5]. The overall algorithm can be summarized as follows:

1. Get the position $(x, y)$ of finger touch on the touchscreen from the digitizer of the tablet device.

2. Compute the distance (vertical and horizontal) to the bezel.

3. If the distance is smaller than the distance threshold $\delta$, then estimate an input voltage from the equation above.

4. If the distance is larger than the distance threshold $\delta$, then put a maximum input voltage.

5. Send the amplified input signal (a two-peak pulse) to piezoelectric actuators.

6. Repeat Steps 1 through 5.

A pseudo code of the algorithm is described in Figure 6.

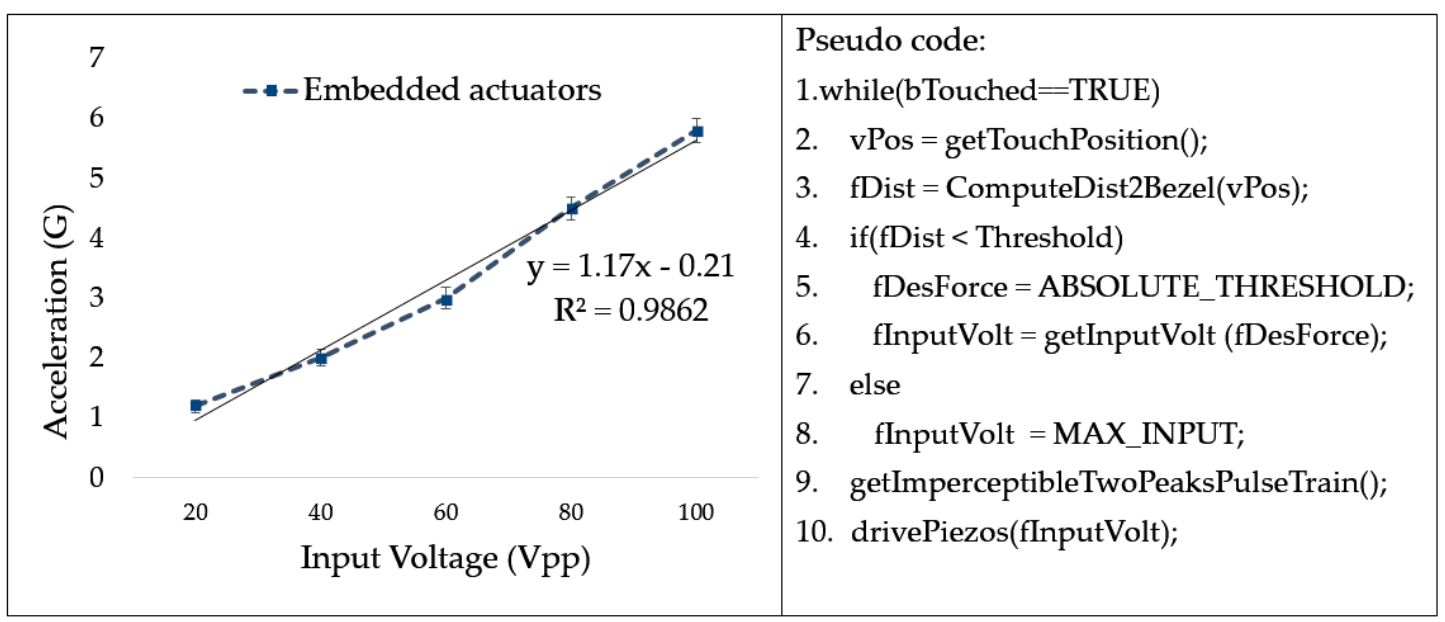

Figure 6. A proposed algorithm to equalize tactile key click effects on the table touchscreen; a linear regression function that has the best fit to the data points (embedded actuators) and a pseudo code that implements the key click equalization algorithm (right).

The implemented algorithm equalizing the strength of tactile feedback on the touchscreen was tested and compared with the measured force distributions generated from four actuators. Two actuators were attached next to the home button, and the other two actuators were located at the middle of two vertical sides, respectively. The same accelerometer was used for measuring tactile 
force feedback on the touchscreen. Accelerations at sixteen points equally spaced on the touchscreen were measured when all of the piezoelectric actuators were bent at the same time. Then, the measured values were graphically visualized in Figure 7 . In the plots, the redder is the stronger tactile feedback. It was obvious from the results that the force distribution was greatly improved after applying our proposed global equalization method.

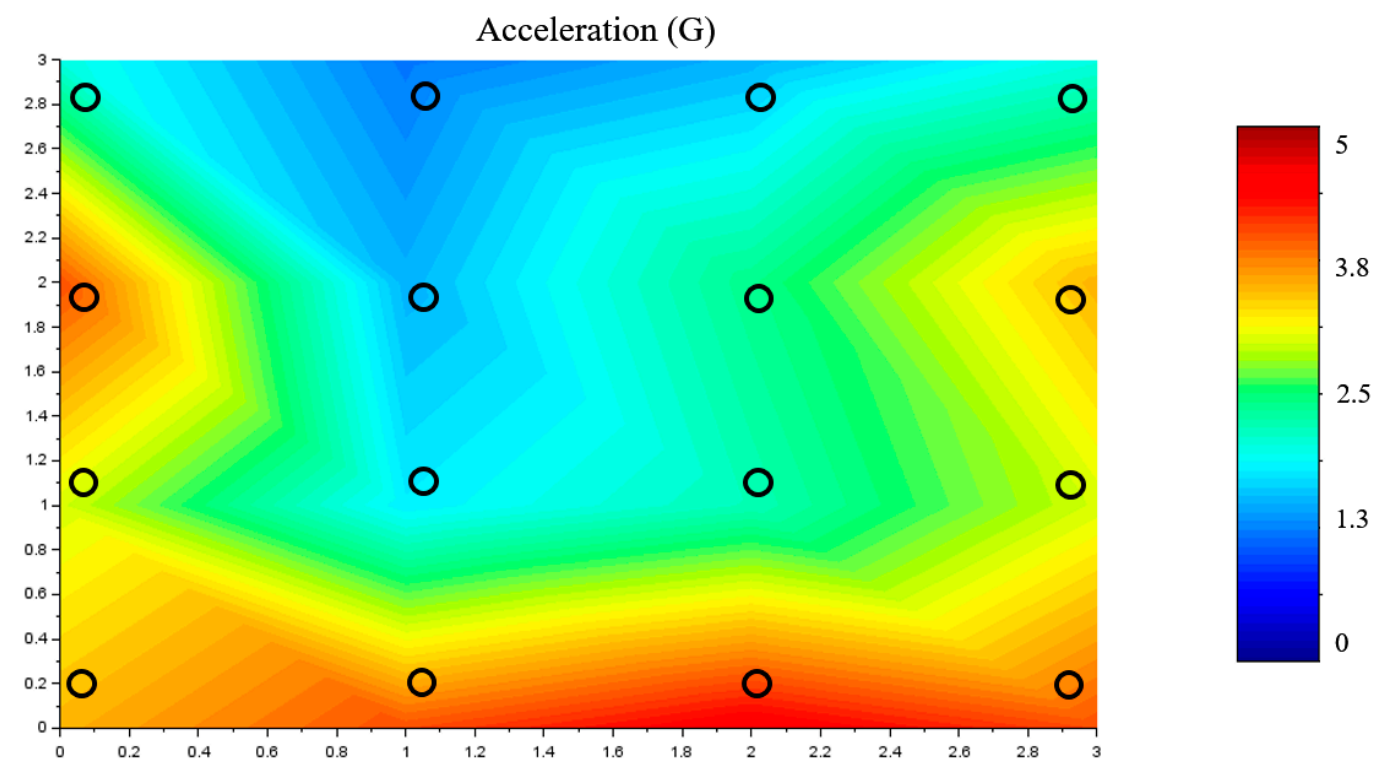

(a)

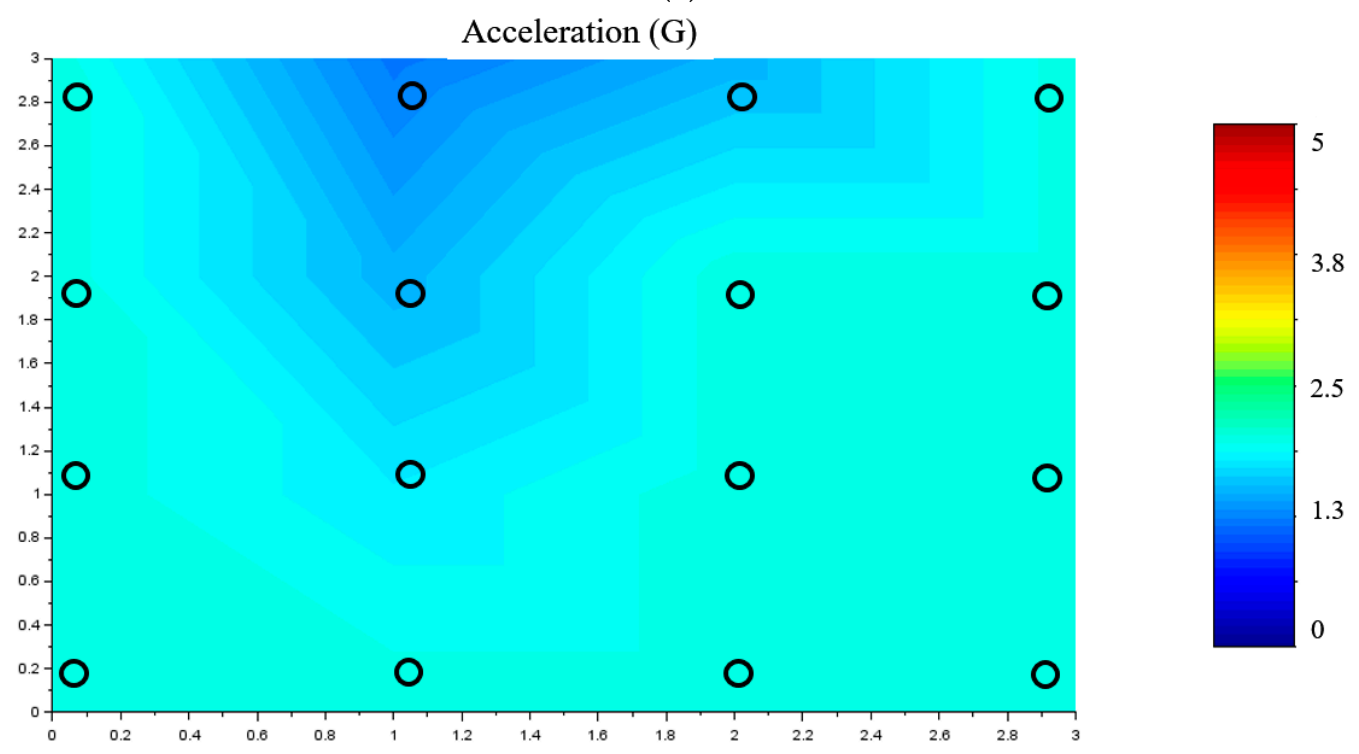

(b)

Figure 7. Accelerations distributed on a 10.1 inch commercial touchscreen (Samsung Galaxy Tab); non-uniform tactile feedback distribution (a) and the globally equalized distribution by our proposed algorithm (b). Note that the redder area means the stronger tactile feedback, and black circles are points where accelerations were measured.

\section{Results}

Three evaluation experiments were conducted to verify the piezoelectric actuators designed with optimum parameters (three layers and the layer ratio) and the haptic discrimination threshold and to measure typing performance with the tactile feedback system. A piezoelectric actuator manufactured 
based on the final design was tested on a 10.1 inch touchscreen under a laboratory environment. The strength of vibro-tactile feedback (acceleration) on a commercial touchscreen was measured as increasing input voltage to a piezoelectric actuator. Accelerations were measured with two conditions, free stroke on a detached touchscreen and after embedding the actuator in a 10.1 inch tablet. For the free stroke condition, the piezo actuator was glued on a separated touch glass (see Figure 8), and for the embedding condition, the actuator was attached to the bezel space between the LCD panel and the touchscreen of a tablet. Then, an accelerometer (KISTLER 8688A50) was placed on the touchscreen to measure accelerations as the input voltage (a square wave of $500 \mathrm{~Hz}$ ) of the piezo actuator was incrementally altered. The input signal to the piezo was controlled by a function generator (Agilent 33120). Figure 8 shows the accelerations measured with the two conditions (before and after embedding actuators). The results showed that, for the embedding condition, the strength of tactile feedback was reduced by $27.3 \%$ due to piezoelectric actuators' compression by the process of reassembly of the tablet device.

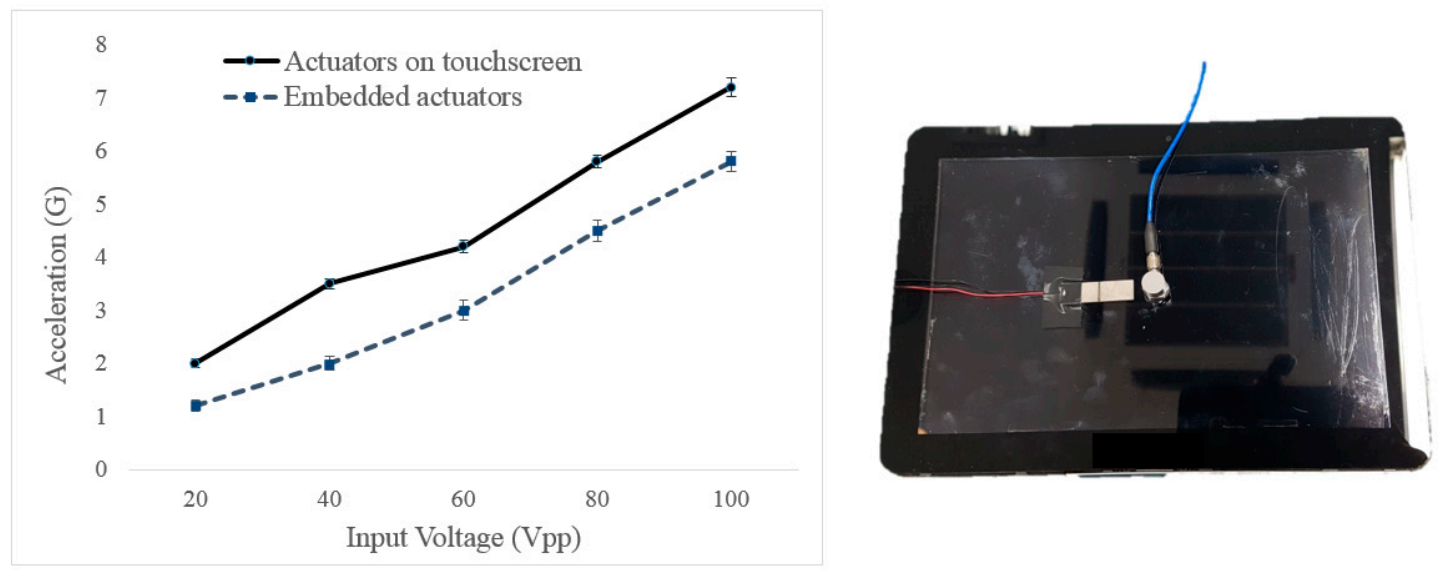

Figure 8. Acceleration values (left) measured on a 10.1 inch touchscreen with a three-layer piezoelectric actuator as a function of input voltage (Vpp) for two conditions, free stroke on a detached touchscreen (right) and after embedding the actuators in a tablet.

The second experiment was conducted to verify whether the two-peak tactile pulse made users feel better about the touchscreen. For this experiment, ten participants (five males and five females, age range 21-29, average age 25.5 years old) who had never experienced haptic interfaces took part in the experiment. The experiment setup was the same as shown in Figure 4, but the value of $t$ of the test stimulus in Figure 3 a was set to $3 \mathrm{~ms}$, which was much smaller than the estimated discrimination threshold $(3.919 \pm 0.393)$ to ensure imperceptibility. The test and the reference stimuli were randomly displayed to avoid subjects' expectation errors. No sound feedback was provided during the experiment. The participant's task was to indicate which one felt better as a key click. Each participant was tested ten times for both stimuli, resulting in a total of ten trials per participant. It took about five minutes for each participant to complete all of the trials. The result was that $81 \%$ of participant responses were that the two-peak pulse was better, but there was a variation from person to person due to different sensitivities.

The last experiment was conducted to evaluate typing performance (efficiency, speed, and accuracy) with the developed tactile touchscreen for a 10.1 inch tablet device. For the experiment, a commercial QWERTY keyboard was implemented on the Android platform of a tablet device (uPad), as shown in Figure 9. The developed system had a latency of $1.87 \mathrm{~ms}$ from sensing touch using the Android touch event listener to tactile feedback. A two-peak tactile pulse ( $t=3 \mathrm{~ms})$ was used for tactile key click feedback, and the signal was amplified to 65 Vpp by the amplifier (TI DRV 2667, gain $38.4 \mathrm{~dB}$ ). The tactile feedback was synchronized with the key-down event provided from the Android platform. Well-known metrics, key strokes per character (KSPC), words per minute (WPM), and uncorrected error rate (UER) $[1,17,22]$, were used for quantitatively measuring typing performance 
across two conditions, visual (V) only and visual and haptic (VH), to see the effectiveness of tactile feedback when adding to an existing soft keyboard. The two conditions were counterbalanced and randomized. For the $\mathrm{V}$ condition, the pressed key was highlighted in white, while tactile feedback was additionally provided for the $\mathrm{VH}$ condition. The experiment consisted of ten blocks per condition, and each block had fifteen sentences that were randomly selected from a phrase dataset created by [23]. Each sentence was displayed in red at the top of the touchscreen, as seen in Figure 9, until the user finished transcribing the displayed sentence. The white text box showed what the user typed in real time. Ten participants (six males and four females, average 24.2 years old) took part in the experiment. All participants were skilled typists and had at least two years of experiences using the soft keyboard of a 10.1 inch tablet device. Before starting the experiment, a training session (about 5-10 min) was provided to participants to get familiar with the tactile soft keyboard. Each participant was then instructed to transcribe displayed sentences as quickly and accurately as possible. The participants wore earplugs to block noise during the experiment. It took about fifty minutes for each participant to complete the experiment.
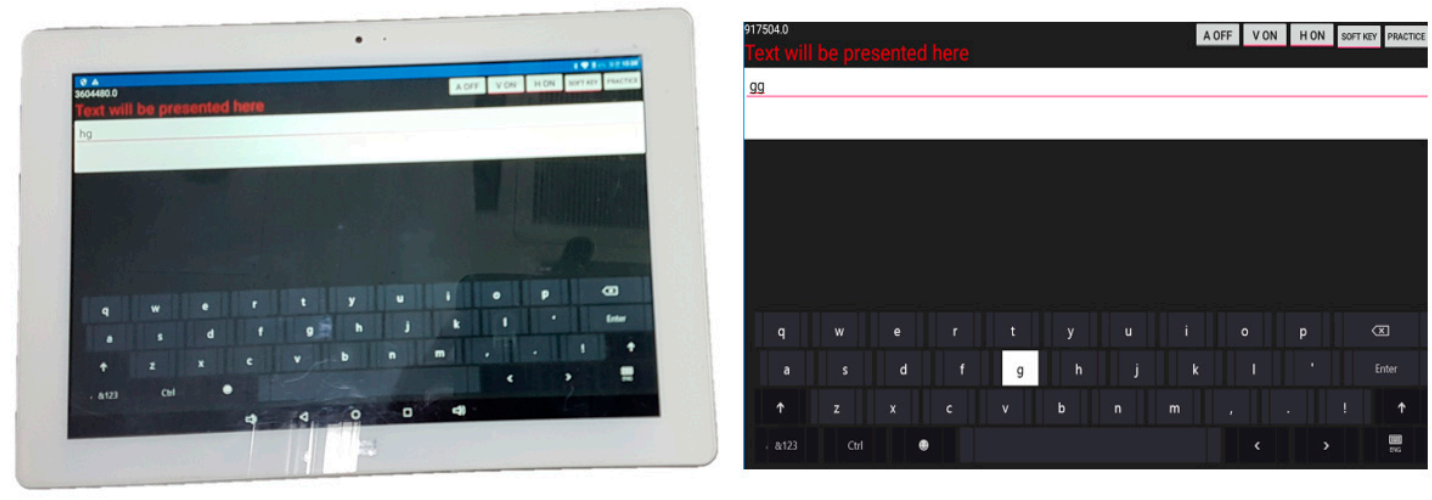

Figure 9. Soft keyboard design and a user interface implemented on the Android platform with a 10.1 inch commercial touchscreen ( $\mathrm{uPad}$ ) for a user study. The white text field was an area for a user to transcribe a red sentence randomly selected from a test set of sentences created by [23]. For the visual (V) condition, the pressed key was highlighted in white, while tactile key click feedback was additionally provided for the visual and haptic $(\mathrm{VH})$ condition.

Figure 10 shows the results of typing performance (KSPC, WPM, UER) tested with the haptic soft keyboard. The bars represent the average values of each metric obtained from the experiment, and the error bars show the standard deviations. Note that a lower value meant better performance, except with WPM, which was interpreted as typing speed. As an efficiency index, KSPC ranged from 1.17 to 1.38 , with $\mathrm{VH}$ appearing to be more efficient, which showed better productivity when tactile feedback was added. The average UERs, typing errors, were 0.54 and 0.8 for $\mathrm{VH}$ and $\mathrm{V}$, respectively, which demonstrated improved accuracy with tactile feedback. In contrast, there was no difference in typing speed observed from the average WPM values from 28.4 and 28.9 for $\mathrm{V}$ and $\mathrm{VH}$, respectively. A one-way ANOVA showed that there was no significant difference $(p>>0.05)$ across conditions for the three metrics. 


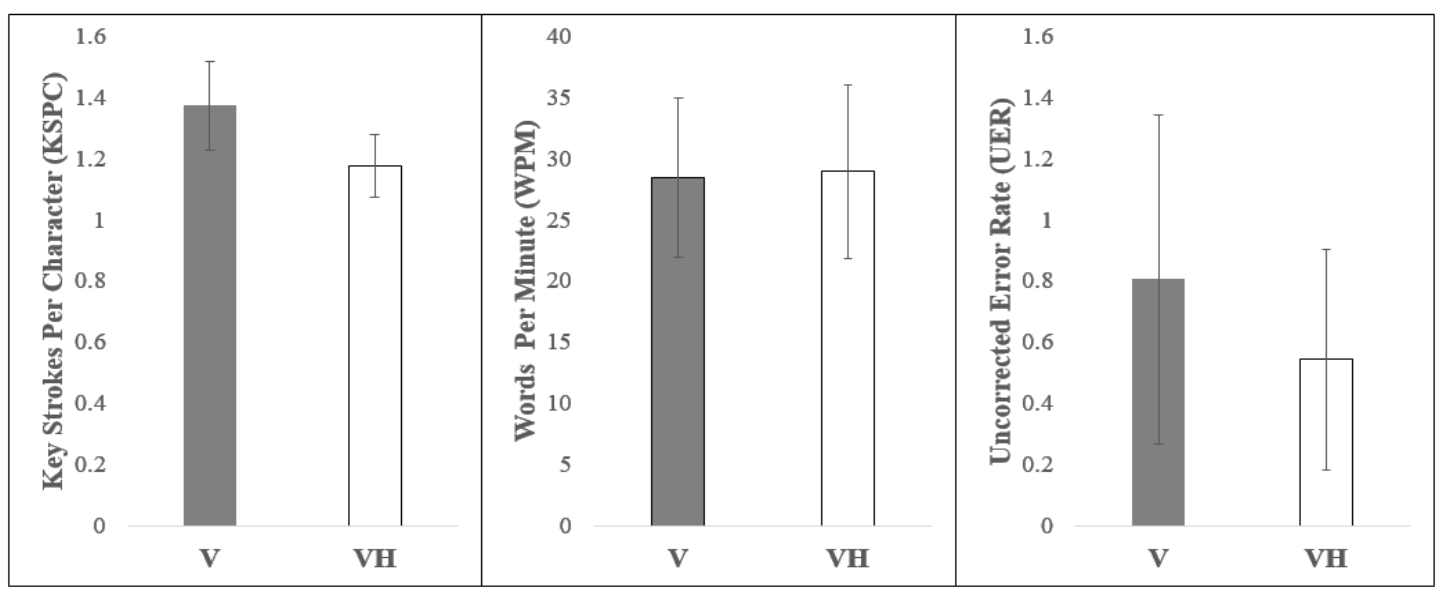

Figure 10. Typing performance results measured by a user study across two conditions $\mathrm{V}$ and $\mathrm{VH}$. Key strokes per character (KSPC), words per minute (WPM), and uncorrected error rate (UER) were interpreted as typing efficiency, speed, and accuracy on the 10.1 inch touchscreen. The bars represent means and standard deviations of each metric for the two conditions. A lower value meant better performance except for WPM.

\section{Discussion}

In the present study, we investigated an effective way of providing a stronger tactile feeling of key click on tablet sized touchscreens. It is imperative because deformation of the larger touchscreen can be achieved by the stronger piezoelectric bender. It is known by Equation (4) that the thickness of a ceramic layer is inversely proportional to mechanical displacement, which justifies the need for multi-layer piezoelectric actuators that require more expensive manufacturing cost and current because the capacitance increases with the number of layers. For this reason, finding optimized parameters (the number of layers and the layer ratio) is crucial, especially for mobile devices that have limited power and space to add actuators. The best parameters according to the simulations were three layers and 1.4 as the ratio of the internal ceramic layer to the external ceramic layer. It was also found from pilot experiments that the bending performance was better when the poles of the three layers were connected in a series, as seen in Figure 2. The bending performance of the manufactured actuator was tested by measuring accelerations. The results from Figure 8 demonstrate the sufficient strength to simulate key clicks on a 10.1 inch touchscreen with low voltage by referring to the detection threshold $(1.14 \mathrm{G})$ on a commercial touchscreen [1]. However, it was also found that there was a significant drop (average $27.3 \%$ ) of tactile force display on the touchscreen due to the compression force caused by reassembling the tablet device after bonding the actuators. This issue could be resolved by designing a new internal structure that preserves the mechanical bending whenever voltage is applied to piezoelectric actuators.

In addition, the discrimination thresholds (JNDs) measured in this study to understand the sensitivity to time differences of a tactile pulse or a tactile pulse combined with sound were the first results ever reported for 10.1 inch commercial touchscreens. The discrimination threshold for the haptics only condition was used as an upper bound perception value to increase the perceptual strength of a tactile key click effect. The HA threshold can be a guideline for designing a multimodal feedback signal commonly used in mobile devices. Through this approach, users can surely feel the stronger tactile feedback on 10.1 inch touchscreens, and the use of mobile battery driving multiple actuators can also be reduced, since an imperceptible two-peak pulse consumes less energy than a one-long-peak pulse. The desired acceleration value on the touchscreen was also determined by taking into account the perception data (detection threshold $1.17 \mathrm{G}$ ) estimated from [2] for an algorithm equalizing the distribution of tactile forces, as shown in Figure 6. This study shows a practical example of how to employ human perception data, but further studies are needed to apply various form factors in terms of the size and the material of touchscreens. Another contribution of this study is the proposal of a new algorithm that can equalize the distribution of force feedback on a 10.1 inch touchscreen. Due to the 
material characteristics of 10.1 inch commercial touchscreens, vibro-tactile signals are not transparently delivered to the center from the bezel, where multiple piezoelectric actuators are bonded. People feel uncomfortable near the bezel if the tactile force is not uniformly distributed. Our proposed algorithm improves the force distribution by adaptively reducing the force values to the distance of the bezel, but further study is needed to improve the upper area that still appeared relatively blue, as visualized Figure 7.

Finally, the developed tactile feedback system was verified by measuring typing performance using well-known metrics, KSPC, WPM, and UER, which represented typing efficiency (key strokes per character), typing speed, and accuracy (uncorrected errors), respectively. The results demonstrated that typing efficiency and accuracy were improved with tactile feedback, whereas there was no difference in typing speed; however, there were no statistically significant differences between the two conditions due to different typing skills from naïve to skilled typists. Note that our previous study showed that tactile feedback was more effective with skilled typists []. Several factors may have contributed to this result. First, typing confirmation with tactile feedback played an important role in reducing typing errors or correcting errors, because skilled typists tended to press keys without looking at each key character, which resulted in higher KSPCs and UERs with the V condition. Second, a time delay in the tactile feedback for confirming key clicks caused lower typing speed with the VH condition. The time delay often resulted in unsynchronized tactile feedback, especially with fast typists. Third, typing speed may have been affected by learning during the experiment. It is possible that participants did not show good performance in typing speed since they were still getting used to the tactile feedback condition by the end of the experiment. This could be proved by a further study investigating the effect of tactile feedback with a longer time of training.

Author Contributions: The first author (S.Y.) and the corresponding author (K.K.) developed algorithms and the piezo driving circuit system, and the second author (S.H.J.) verified and analyzed the research results. The corresponding author (K.K.) guided the research direction. All authors made substantial contributions in the writing and revision of the paper.

Funding: This work was supported by Incheon National University Research Grant in 2016 (Grant No. 2016).

Conflicts of Interest: The authors declare no conflict of interest.

\section{References}

1. Han, B.; Kim, K. Typing performance evaluation with multimodal soft keyboard completely integrated in commercial mobile devices. J. Multimodal User Interfaces 2015, 9, 173-181. [CrossRef]

2. Kim, K. Perception-based tactile soft keyboard for the touchscreen of tablets. Mob. Inf. Syst. 2018, $2018,9$. [CrossRef]

3. Findlater, L.; Wobbrock, J.O.; Wigdor, D. Typing on flat glass: Examining ten-finger expert typing patterns on touch surfaces. In Proceedings of the SIGCHI Conference on HUMAN Factors in Computing Systems, Vancouver, BC, Canada, 7-12 May 2011; pp. 2453-2462.

4. Findlater, L.; Wobbrock, J. Personalized input: Improving ten-finger touchscreen typing through automatic adaptation. In Proceedings of the SIGCHI Conference on Human Factors in Computing Systems, Austin, TX, USA, 5-10 May 2012; pp. 815-824.

5. Li, F.C.Y.; Guy, R.T.; Yatani, K.; Truong, K.N. The 1line keyboard: A QWERTY layout in a single line. In Proceedings of the 24th annual ACM symposium on User interface software and technology, Santa Barbara, CA, USA, 16-19 October 2011; pp. 461-470.

6. Goel, M.; Jansen, A.; Mandel, T.; Patel, S.N.; Wobbrock, J.O. ContextType: Using hand posture information to improve mobile touch screen text entry. In Proceedings of the SIGCHI Conference on Human Factors in Computing Systems, Paris, France, 27 April-2 May 2013; pp. 2795-2798.

7. Jo, S.M.; Cho, S.B. A Personalized Context-aware Soft Keyboard adapted by Random Forest trained with Additional Data of Same Cluster. Neurocomputing 2019, 353, 17-27. [CrossRef] 
8. Poupyrev, I.; Mauyama, S. Tactile interfaces for small touch screens. In Proceedings of the 16th Annual ACM Symposium on User Interface Software and Technology, Vancouver, BC, Canada, 2-5 November 2003; pp. 217-220.

9. Rabin, E.; Gordon, A.M. Tactile feedback contributes to consistency of finger movements during typing. Exp. Brain Res. 2004, 155, 362-369. [CrossRef] [PubMed]

10. Brewster, S.; Brewster, S.; Chohan, F.; Brown, L. Tactile feedback for mobile interactions. In Proceedings of the SIGCHI Conference on Human Factors in Computing Systems, San Jose, CA, USA, 28 April-3 May 2007; pp. 159-162.

11. Hoggan, E.; Brewster, S.A.; Johnston, J. Investigating the effectiveness of tactile feedback for mobile touchscreens. In Proceedings of the SIGCHI Conference on Human Factors in Computing Systems, Florence, Italy, 5-10 April 2008; pp. 1573-1582.

12. Koskinen, E.; Kaaresoja, T.; Laitinen, P. Feel-Good Touch: Finding the Most Pleasant Tactile Feedback for a Mobile Touch Screen Button; ACM: New York, NY, USA, 2008.

13. Jansen, Y.; Karrer, T.; Borchers, J. MudPad: Localized Tactile Feedback on Touch Surfaces. In Proceedings of the 23nd annual ACM symposium on User interface software and technology, New York, NY, USA, 3-6 October 2010; ACM: New York, NY, USA, 2010; pp. 385-386.

14. Hoffmann, A.; Spelmezan, D.; Borchers, J. TypeRight: a keyboard with tactile error prevention. In Proceedings of the SIGCHI Conference on Human Factors in Computing Systems, Boston, MA, USA, 4-9 April 2009; pp. 2265-2268.

15. McAdam, C.; Brewster, S. Distal tactile feedback for text entry on tabletop computers. In Proceedings of the 23rd British HCI Group Annual Conference on People and Computers: Celebrating People and Technology, Cambridge, UK, 1-5 September 2009; pp. 504-511.

16. Kaaresoja, T.; Anttila, E.; Hoggan, E. The effect of tactile feedback latency in touchscreen interaction. In Proceedings of the 2011 IEEE World Haptics Conference, Istanbul, Turkey, 21-24 June 2011; pp. 65-70.

17. Han, B.K.; Kim, K.; Yatani, K.; Tan, H.Z. Text entry performance evaluation of haptic soft QWERTY keyboard on a tablet device. In Proceedings of the International Conference on Human Haptic Sensing and Touch Enabled Computer Applications, Versailles, France, 24-26 June 2014; Springer: Berlin/Heidelberg, Germany, 2014; pp. 325-332.

18. Jeon, S.; Lee, H.; Jung, J.; Kim, J.R. User-Adaptive Key Click Vibration on Virtual Keyboard. Mob. Inf. Syst. 2018, 2018, 1-9. [CrossRef]

19. Wang, Q.M.; Cross, L.E. Constitutive equations of symmetrical triple layer piezoelectric benders. IEEE Trans. Ultrason. Ferroelectr. Freq. Control 1999, 46, 1343-1351. [CrossRef] [PubMed]

20. Levitt, H. Transformed up-down methods in psychoacoustics. J. Acoust. Soc. Am. 1971, 49, 467-477. [CrossRef]

21. Goldstein, E.B. Sensation and Perception, 9th ed.; Cengage Learning: Boston, MA, USA, 2014.

22. Soukoreff, R.W.; Mackenzie, I.S. Metrics for text entry research: An evaluation of MSD and KSPC, and a new unified error metric. In Proceedings of the SIGCHI Conference on Human Factors in Computing Systems, Lauderdale, FL, USA, 5-10 April 2003; pp. 113-120.

23. Mackenzie, I.S.; Soukoreff, R.W. Phrase sets for evaluating text entry techniques. In Proceedings of the $\mathrm{CHI}^{\prime} 03$ Extended Abstracts on Human Factors in Computing Systems, Ft. Lauderdale, FL, USA, 5-10 April 2003; ACM: New York, NY, USA, 2013; pp. 754-755.

(C) 2019 by the authors. Licensee MDPI, Basel, Switzerland. This article is an open access article distributed under the terms and conditions of the Creative Commons Attribution (CC BY) license (http://creativecommons.org/licenses/by/4.0/). 